\title{
The Impact of COVID-19 on Pediatric Telepsychiatry Training in Child and Adolescent Psychiatry Fellowships
}

\author{
Sandra M. DeJong ${ }^{1}\left[\right.$ Deborah Brooks ${ }^{2} \cdot{\text { Shabana } \mathrm{Khan}^{3} \cdot \text { Samantha Reaves }^{2} \cdot \text { Bianca Busch }^{4} \cdot \text { Dan Alicata }}^{5}$. \\ Ujjwal Ramtekkar ${ }^{6} \cdot$ Lan Chi Vo $^{7} \cdot$ David Pruitt $^{2}$
}

Received: 9 March 2021 / Accepted: 2 November 2021 / Published online: 2 December 2021

(c) Academic Psychiatry 2021

\begin{abstract}
Objective This report summarizes findings from a 2020 survey of US child and adolescent psychiatry training programs that explored the impact of the COVID-19 pandemic on pediatric telepsychiatry training. The authors hypothesized that telepsychiatry training significantly increased during the pandemic, in part due to legal and regulatory waivers during the COVID-19 public health emergency.

Methods In August 2020, an anonymous, 28-question online survey was emailed to all (138) accredited child psychiatry fellowships on the Accreditation Council for Graduate Medical Education website. Forty-nine programs responded (36\%). This analysis focuses on three of the 28 questions relevant to the hypotheses: characteristics of the program's training in telepsychiatry; perceived impediments to clinical training; and perceived impediments to didactic training pre-COVID onset vs. post-COVID onset, respectively. Total scores were created to investigate differences in training programs and impediments to including telepsychiatry pre- and post-COVID onset. Paired sample $t$-tests were used to compare means pre- and post-COVID onset.

Results Results provided support for significant differences between training components related to telepsychiatry pre- and post-COVID onset, with participants reporting more training components post-COVID onset $(M=5.69)$ than pre-COVID onset $(M=1.80) ; t(48)=9.33, p<.001$. Participants also reported significantly fewer barriers to providing clinical experiences in pediatric telepsychiatry post-COVID onset $(M=2.65)$ than pre-COVID onset $(M=4.90) ; t(48)=-4.20, p<.001$. Conclusions During the COVID-19 pandemic, pediatric telepsychiatry training in child psychiatry fellowships increased significantly. Perceived barriers to providing clinical, but not didactic, training decreased significantly.
\end{abstract}

Keywords Telepsychiatry $\cdot$ Pediatric $\cdot$ Child $\cdot$ Training $\cdot$ COVID-19

Sandra M. DeJong

sdejong@cha.harvard.edu

1 Cambridge Health Alliance, Harvard Medical School, Cambridge, MA, USA

2 University of Maryland School of Medicine, Baltimore, MD, USA

3 New York University (NYU) Grossman School of Medicine, New York, NY, USA

4 Boston Children's Hospital, Harvard Medical School, Boston, MA, USA

5 University of Hawaii at Manoa, John A. Burns School of Medicine, Honolulu, HI, USA

6 Nationwide Children's Hospital, The Ohio State University, Columbus, OH, USA

7 Children's Hospital of Philadelphia, University of Pennsylvania School of Medicine, Philadelphia, PA, USA
The USA currently faces a shortage of child and adolescent psychiatrists. While the estimated need is 48 to 49 thousand based on the epidemiology of psychiatric disorders in children, only 8 to 9 thousand child psychiatrists are in practice nationally; most counties do not have a child psychiatrist [1-3]. The COVID-19 pandemic has led to an increased demand for mental health services for youth, exacerbating the existing shortage [4].

The literature on telepsychiatry education and training is limited [5, 6], and the majority does not address considerations unique to pediatric telepsychiatry [7]. A literature review on telepsychiatry in graduate medical education identified 20 relevant publications describing training psychiatry residents in telepsychiatry [8]. Most of the literature was primarily descriptive, and the authors concluded that 
a more evidence-based approach to telepsychiatry training is needed.

Pediatric telepsychiatry is defined as direct or consultative patient care to youth and families provided remotely through audio/video-conferencing platforms. It offers an opportunity to improve access to pediatric behavioral healthcare through both direct care and consultation to healthcare and nonhealthcare settings (e.g., schools). Its effectiveness with children and youth has been demonstrated [9]. Despite the availability of telehealth since the 1950s, uptake of telepsychiatry by child psychiatrists has historically been slow [10]. In a survey of child psychiatry fellowship programs in May 2019 (response rate 35\%), 35\% of respondents reported offering no telepsychiatry clinical experience in their program, 46\% reported "some," and only 19\% reported "a lot" [11]. Sixty percent of respondents had no formal didactic curriculum.

The 2019 survey and other reports suggest that real and perceived hurdles such as the Ryan Haight Act, medical licensing issues, lack of reimbursement, and restrictions regarding clinical settings have prevented this uptake [12]. Lack of faculty expertise and educational resources specific to child psychiatry has also presented obstacles [10]. However, when the COVID-19 pandemic erupted in March 2020 in the USA, a Public Health State of Emergency temporarily waived many of the regulatory restrictions (with some variation between states), and training programs and clinical services had to rapidly implement telepsychiatry.

With this rapid transition to remote healthcare delivery, child psychiatry services - seemingly overnight-were delivered via platforms such as Zoom ${ }^{\mathrm{TM}}$ and Google Meet ${ }^{\mathrm{TM}}$ to patients wherever they were living. Due to the public health emergency, new federal changes were announced by the Office for Civil Rights (OCR) of the Department of Health and Human Services. This notice allowed covered healthcare providers subject to the regulatory requirements under the Health Insurance Portability and Accountability Act (HIPAA) to provide telehealth services using technologies that may not fully comply with the requirements of the HIPAA rules without penalty [13]. As a result, use of nonpublic facing applications such as Facetime ${ }^{\mathrm{TM}}$ and Skype ${ }^{\mathrm{TM}}$ was deemed acceptable at the federal level. In addition, the Centers for Medicare and Medicaid Service expanded telehealth reimbursement for a wide range of behavioral health services delivered via telephone, thus removing the major barrier for use of telephone as a primary service modality [14]. Similarly, the Drug Enforcement Administration (DEA) announced changes in policies to allow DEA-registered providers to prescribe controlled substances without an in-person examination [15]. At the state level, licensure requirements were adjusted to allow care delivery across the state lines for established patients. (There has been significant variability among states in allowing out-of-state providers to deliver care, as well in permitting in-state providers to care for patients residing out-of-state).

This study was designed to assess the impact of these clinical and regulatory changes on telepsychiatry training in child psychiatry fellowships. The hypothesis was that these changes in clinical service delivery and the loosening of previous restrictions led to a significant increase in pediatric telepsychiatry training. A secondary hypothesis was that the type of service provided changed to more "directto-consumer" care (patients in their homes).

\section{Methods}

The 28-item survey was launched in August 2020, about 6 months into the pandemic. (A copy of the survey is available upon request). It was distributed online to all child psychiatry training programs accredited by the Accreditation Council on Graduate Medical Education (ACGME, 138) using Qualtrics ${ }^{\mathrm{TM}}$ software (Qualtrics, Provo, UT). It was open for 4 weeks with weekly reminders, and Institutional Review Board approval was waived by the Cambridge Health Alliance Institutional Review Board. Results were anonymously collected and collated. The questions focused on amount of time, perceived importance, types of clinical experiences, didactic curricula, and perceived impediments to providing clinical and didactic training in pediatric telepsychiatry. Comment boxes after each topic allowed for qualitative responses which were not formally analyzed. Three questions related to characteristics of the training program.

The current analysis focuses on 3 out of the 28 questions: question 7, which focused on characteristics of their training in telepsychiatry pre- vs. post-COVID onset; question 9, which asked programs about impediments to clinical training they faced pre- vs. post-COVID onset; and question 11, which asked programs about impediments to didactic teaching they faced pre- vs. post-COVID onset.

Total scores were created to investigate differences in training programs and impediments to incorporating telepsychiatry pre- and post-COVID onset. Eleven items were used to describe components of pediatric telepsychiatry training (i.e., dedicated telepsychiatry rotations, supervisors with dedicated time and expertise in telepsychiatry, consultation to offsite mental health providers etc.). Participants endorsed the presence/absence of each component (yes/no) in the past (pre-COVID onset) and currently (post-COVID onset). These dichotomous scores were combined to create a total score out of 11 .

Thirteen items were used to assess impediments to telepsychiatry clinical experiences (i.e., limited number of faculty with time to supervise the experiences, limited time in the clinical curriculum, insufficient clinical space at our 
site etc.). Participants endorsed the presence/absence of each impediment (yes/no) in the past (pre-COVID onset) and currently (post-COVID onset). These dichotomous scores were combined to create a total score out of 13.

Seven items were used to assess impediments to providing, improving, expanding a didactic curriculum in pediatric telepsychiatry (i.e., limited number of faculty with time to design the curriculum, insufficient funding for faculty time and other resources, graduates of our program are unlikely to be practicing pediatric telepsychiatry, etc.). Participants endorsed the presence/absence of each barrier (yes/no) in the past (pre-COVID onset) and currently (post-COVID onset). These dichotomous scores were combined to create a total score out of 7 .

All three questions had comment boxes for qualitative responses.

To investigate changes in ratings of participant's training components and barriers to implementing telehealth in clinical experiences and didactic programs, paired sample $t$-tests were used to compare means pre- and post-COVID onset. A Bonferroni correction was used to account for multiple tests $(3 ; p<0.016)$.

\section{Results}

See Table 1 for a summary of paired sample $t$-test and effect size estimates for training components and impediments to clinical vs. didactic training pre- and post-COVID onset. See Table 2 for a summary of differences between each of the items for the three questions analyzed here.

The survey response rate was $49 / 138$ or $36 \%$. Responding programs were primarily academic $(34 \%, 40)$ and urban $(16 \%, 19)$. Approximately $14 \%$ (16) described themselves as underserved and community-based (17). Most programs were small, defined as $2-3$ fellows per class $(54 \%, 25)$, and older than 15 years $(76 \%, 35)$. The geographic distribution was relatively equal, with a preponderance of programs in the southeast $(30 \%, 14)$.

Results provided support for significant differences between the presence of training components related to telepsychiatry pre- and post-COVID onset, with participants reporting more training components post-COVID onset
$(M=5.69)$ than pre-COVID onset $(M=1.80) ; t(48)=9.33$, $p<0.001$. Participants also reported significantly fewer barriers to providing clinical experiences in pediatric telepsychiatry post-COVID onset $(M=2.65)$ than preCOVID onset $(M=4.90) ; t(48)=4.20, p<0.001$. 00. This finding suggests an association between the increase in clinical training in pediatric telepsychiatry and a reduction in barriers to providing clinical telepsychiatry experiences. As one respondent put it, "COVID has completely changed the adoption of this innovation."

Participants did not report significant changes in the barriers to providing, improving, or expanding the didactic program in pediatric telepsychiatry post-COVID onset $(M=1.78)$ than pre-COVID onset $(2.08) ; t(48)=-1.29$, $p>0.016$. This finding suggests there were few barriers to providing, improving, or expanding didactics programs both pre- and post-COVID onset. Hedge's $g$ was used to estimate the effect size. Results suggested there was a small effect on participant's rating of their programs telepsychiatry components and impediments to clinical experiences when considering COVID-19.

Table 2 provides detailed information about participants' responses to individual items for each of the three areas to further explore differences in training program components and impediments to clinical experiences and training pre- and post-COVID onset. Seven times as many programs reported providing direct care to patients in their homes since the beginning of the pandemic (pre $-n=6$ vs. post $-n=42$ ). In most training program areas (e.g., dedicated telepsychiatry rotations, including formal didactics), twice as many programs reported having individual training components than pre-COVID onset. Additionally, half the number of programs reported legal and regulatory hurdles (pre-31 vs. post-14) and faculty interest in developing expertise as an impediment to clinical experiences $(Z=-3.55,0.000)$, and insufficient funding for faculty time and other resources as an impediment to providing didactics in pediatric telepsychiatry (pre-15 vs. post-6) as barriers to their telepsychiatry programs. This finding suggests there are more reported training program components and fewer barriers to telepsychiatry clinical experiences post-COVID onset for the participants $(\mathrm{Z}=-2.71,0.007)$.

Table 1 Paired sample $t$-test and effect size estimates for training experiences and impediments in clinical and didactic training

\begin{tabular}{|c|c|c|c|c|c|c|c|c|}
\hline & \multicolumn{2}{|c|}{ Pre-COVID onset } & \multicolumn{2}{|c|}{ Post-COVID onset } & \multirow[t]{2}{*}{$t$} & \multirow[t]{2}{*}{$d f$} & \multirow[t]{2}{*}{$p$} & \multirow[b]{2}{*}{ Hedge's $g$} \\
\hline & Mean & $\begin{array}{l}\text { Standard } \\
\text { deviation }\end{array}$ & Mean & $\begin{array}{l}\text { Standard } \\
\text { deviation }\end{array}$ & & & & \\
\hline Training Experiences (clinical and didactic) & 1.80 & 2.71 & 5.69 & 3.17 & -9.33 & 48 & .000 & 2.94 \\
\hline Impediments to clinical experiences & 5.10 & 3.78 & 2.71 & 2.26 & 4.20 & 48 & .000 & 4.01 \\
\hline Impediments to didactics & 2.16 & 2.02 & 1.84 & 1.72 & 1.29 & 48 & .207 & 1.77 \\
\hline
\end{tabular}


Table 2 Percentage of programs endorsing the presence of training components and impediments to clinical and didactic telepsychiatry experience pre- and post-COVID

\begin{tabular}{|c|c|c|c|}
\hline & $\begin{array}{l}\text { Pre-COVID onset } \\
\% \text { of yes responses }(\#)\end{array}$ & $\begin{array}{l}\text { Post-COVID onset } \\
\% \text { of yes responses (\#) }\end{array}$ & $\begin{array}{l}\text { Difference } \\
\%\end{array}$ \\
\hline \multicolumn{4}{|l|}{ Training experience } \\
\hline 1. Dedicated telepsychiatry rotation(s) & $22.4(11)$ & $53.1(26)$ & 30.7 \\
\hline 2. Taught by supervisors with dedicated time and expertise in telepsychiatry & $24.5(12)$ & $42.9(21)$ & 18.4 \\
\hline 3. Includes formal didactic curriculum & $20.4(10)$ & $53.1(26)$ & 32.7 \\
\hline 4. Offers mentoring by child psychiatrists who are telepsychiatry experts & $24.5(12)$ & $36.7(18)$ & 12.2 \\
\hline 5. Includes consultation to offsite mental health providers & $14.3(7)$ & $46.9(23)$ & 32.6 \\
\hline 6. Includes consultation to nonpsychiatric healthcare providers & $18.4(9)$ & $46.9(23)$ & 28.5 \\
\hline 7. Includes consultation to schools, including residential & $8.2(4)$ & $40.8(20)$ & 32.6 \\
\hline 8. Includes consultation to forensics/juvenile justice & $10.2(5)$ & $26.5(13)$ & 16.3 \\
\hline 9. Includes direct care to patients in their homes & $12.2(6)$ & $85.7(42)$ & 73.5 \\
\hline 10. Includes being taught, trained, and/or supervised virtually and remotely & $16.3(8)$ & $83.7(41)$ & 67.4 \\
\hline 11. Includes learning how to teach, train and supervise virtually and remotely & $8.2(4)$ & $53.1(26)$ & 44.8 \\
\hline \multicolumn{4}{|l|}{ Impediments to clinical experience } \\
\hline 1. $\mathrm{ACGME}^{+}$does not require training in pediatric telepsychiatry & $20.4(10)$ & $6.1(3)$ & -14.3 \\
\hline 2. Legal and regulatory hurdles & $63.3(31)$ & $28.6(14)$ & -34.7 \\
\hline 3. Limited number of faculty with time to supervise the experience & $32.7(16)$ & $16.3(8)$ & -16.4 \\
\hline 4. Limited number of faculty with clinical expertise in pediatric telepsychiatry & $57.1(28)$ & $38.8(19)$ & -18.3 \\
\hline 5. Limited interest by faculty in developing expertise in pediatric telepsychiatry & $36.7(18)$ & $6.1(3)$ & -30.6 \\
\hline 6. Insufficient clinical sites interested in receiving care through telepsychiatry & $30.6(15)$ & $2.0(1)$ & -28.6 \\
\hline 7. Limited time in the clinical curriculum & $32.7(16)$ & $12.2(6)$ & -20.5 \\
\hline 8. Insufficient start-up funding & $40.8(20)$ & $18.4(9)$ & -22.4 \\
\hline 9. Insufficient reimbursement for pediatric telepsychiatry services & $57.1(28)$ & $24.5(12)$ & -32.6 \\
\hline 10. Insufficient clinical space at our sites & $14.3(7)$ & $10.2(5)$ & -4.1 \\
\hline 11. Insufficient space at site where patients are at (originating site) & $26.5(13)$ & $18.4(9)$ & -8.1 \\
\hline 12. Insufficient infrastructure/equipment & $44.9(22)$ & $28.6(14)$ & -16.3 \\
\hline $\begin{array}{l}\text { 13. Disparities in patients' access to communication technologies ("the digital } \\
\text { divide") }\end{array}$ & $53.1(26)$ & $61.2(30)$ & 8.1 \\
\hline \multicolumn{4}{|l|}{ Impediments to didactic experience } \\
\hline 1. ACGME does not require teaching of pediatric telepsychiatry & $16.3(8)$ & $14.3(7)$ & -2.0 \\
\hline 2. Limited faculty with time to design curriculum & $44.9(22)$ & $42.9(21)$ & -2.0 \\
\hline 3. Limited number of faculty with expertise in pediatric telepsychiatry & $46.9(23)$ & $42.9(21)$ & -4.0 \\
\hline 4. Insufficient funding for faculty time and other resources & $40.8(20)$ & $40.8(20)$ & 0.0 \\
\hline 5. Limited interest by faculty in developing expertise in pediatric telepsychiatry & $30.6(15)$ & $12.2(6)$ & -18.4 \\
\hline 6. Limited time for additional seminars in the curriculum & $28.6(14)$ & $26.5(13)$ & -2.1 \\
\hline $\begin{array}{l}\text { 7. Graduates of our program are unlikely to be practicing pediatric telepsychia- } \\
\text { try }\end{array}$ & $8.2(4)$ & $4.1(2)$ & -4.1 \\
\hline
\end{tabular}

${ }^{+}$Accreditation Council for Graduate Medical Education

Question 21 of the survey asked respondents to prioritize which regulatory waivers had been most helpful in facilitating the implementation of telepsychiatry. Respondents' top 3 responses were waivers on restrictions on the geographic and originating site (location of patient); reimbursement changes (expansion of billing codes, on-par reimbursement); and waiver of restrictions on prescribing controlled substances. Others noted changes including loss of the facility fee, decreased no-show rate, introduction of telepsychiatry to a wider range of services, and didactics by teleconferencing. But as one respondent commented, "We are still on pins and needles about when plans will stop paying for telehealth with patients at home and for all these waivers to revert." 


\section{Discussion}

The COVID-19 pandemic catalyzed the rapid establishment of telepsychiatry services nationwide, suggesting that clinicians, patients, and systems can rapidly adapt to innovations in telehealth [16-19]. The post-COVID onset survey results indicate that nearly $100 \%$ of the child and adolescent training programs responding integrated telepsychiatry into their requirements, including in education, training, and clinical care across sites and services-inpatient, outpatient, collaborative care, and rural community training sites. One program director stated that telepsychiatry education "should be a competency."

Given the apparent association between the reduction in regulatory impediments and the increased clinical training in pediatric telepsychiatry, reducing the regulatory burden after COVID-19 while thoughtfully shifting to a noncrisis regulatory environment may be important to maintain both clinical training and patient access to remote care [20]. However, as Table 2 indicates, respondents endorsed multiple impediments to providing clinical training, including limited faculty experience, insufficient start-up funding, insufficient reimbursement for services, and inequities in patient access to telehealth. These suggest the need for ongoing faculty development, infrastructure investment, advocacy for improved reimbursement, and addressing technological inequities. One respondent commented, "The digital divide will continue to be a problem until all youth and families have equal access to telehealth."

Efforts to reduce this digital divide might require including space devoted to telehealth access in public institutions such as schools and community centers (although confidentiality is an important potential barrier); advocacy and funding for expanding broadband to lowincome neighborhoods; federal (e.g., Federal Communication Communication) grants for expanding network connectivity in underserved areas; payers distributing devices directly to members; school support for telehealth activities from school-based hardware and software; digital literacy training; and telepsychiatry instructions in various languages for patients and families.

Survey results are limited by the overall response rate of $36 \%$, the higher response rates among academic programs, and the potential for recall bias when responding to the pre-COVID onset questions. The survey itself may have been limited by threats to internal validity as a "homegrown" survey. Survey timing, during the summer and in the middle of COVID-19, may have confounded results. Like all surveys, responses were subject to selection bias. The survey results may not predict trends in pediatric telepsychiatry training as the federal and state-specific regulatory flexibilities and public health emergencies end.
While the survey addressed the quantity of telepsychiatry occurring in child psychiatry programs, the quality of the clinical service being provided by pediatric telepsychiatry across the country is not known. Assuring the confidentiality, privacy, and safety when delivering care to patients' homes; meeting the needs of the youngest and most challenging patients; and ensuring caretaker involvement in the process are a few of the challenges. The field of child psychiatry may need to establish metrics and reinforce quality standards in pediatric telepsychiatry training and clinical care. Such metrics and standards may make it more likely for regulators and payers to maintain certain regulatory and reimbursement flexibilities.

If the current high level of telepsychiatry training in child psychiatry fellowships persists and most programs are comfortable with teaching "the basics," ongoing educational innovations and resource development may be needed. For example, the literature currently lacks teaching resources for working with patients with special considerations (children with sensory deficits, developmental disorders, psychosis, marked hyperactivity, or medical illness) and specific cultural factors. Further teaching resources on conducting play therapy and direct-to-consumer care are also needed.

Funding This work was supported in part by a Faculty Innovation in Education award from the American Board of Psychiatry and Neurology to Dr. DeJong.

\section{Declarations}

Disclosures On behalf of all authors, the corresponding author states that there is no conflict of interest.

\section{References}

1. Substance Abuse and Mental Health Services Administration (SAMHSA). Behavioral Health Workforce Report. 2020. https:// www.samhsa.gov/sites/default/files/behavioral-health-workforcereport.pdf. Accessed 24 Jan 2021.

2. American Academy of Child and Adolescent Psychiatry. 2018. Workforce maps by state. https://www.aacap.org/aacap/Advoc acy/Federal_and_State_Initiatives/Workforce_Maps/Home.aspx. Accessed 25 Jan 2021.

3. American Academy of Child and Adolescent Psychiatry. 2019. Workforce issues. https://www.aacap.org/AACAP/Resources_for Primary_Care/Workforce_Issues.aspx. Accessed 25 Jan 2021.

4. Leeb RT, Bitsko Rh, Rahakrishnan L. Martinez P. Njai R. Holland KM. Mental health-related emergency department visits among children aged $<18$ years during the COVID-19 pandemic - United States, January 1-October 17, 2020. MMWR Morb Mortal Wkly Rep 2020;1675-80. https://www.cdc.gov/mmwr/volumes/69/wr/ mm6945a3.htm. Accessed 11 Jan 2021.

5. Glover JA, Williams E, Hazlett LJ, Campbell N. Connecting to the future: telepsychiatry in postgraduate medical education. Telemed J E Health. 2013;19(6):474-9. 
6. Hoffman P, Kane JM. Telepsychiatry education and curriculum development in residency training. Acad Psychiatry. 2015;39(1):108-9.

7. Khan S, Ramtekkar U. Child and adolescent telepsychiatry education and training. Psychiatr Clin North Am. 2019;42(4):555-62.

8. Sunderji N, Crawford A, Jovanovic M. Telepsychiatry in graduate medical education: a narrative review. Acad Psychiatry. 2015;39(1):55-62.

9. Boydell K, Hodgins M, Pignatiello A, Teshima J, Edwards H, Willis D. Using technology to deliver mental health services to children and youth: a scoping review. J Can Acad Child Adolesc Psychiatry. 2014; 23(2): 87-99. https://www.ncbi.nlm.nih.gov/ pmc/articles/PMC4032077/ . Accessed 25 Jan 2021.

10. Myers K. Advocating for telepsychiatry in psychiatric training. 5 Jan 2018. https://www.psychiatry.org/psychiatrists/practice/telep sychiatry/blog/advocating-for-telepsychiatry-in-psychiatric-train ing. Accessed 25 Jan 2021

11. Khan S, Myers P, Busch B, Brooks D, Alicata D, Ramtekkar U, Vo L-C, DeJong SM. A national pediatric telepsychiatry curriculum for graduate medical education and continuing medical education. J Child Adolesc Psychopharmacol. 2021;31(7):457-63. https:// doi.org/10.1089/cap.2021.0024.

12. Becker CD, Dandy K, Gaujean M, Fusaro M, Scurlock C. Legal perspectives on telemedicine part 1: legal and regulatory issues. Perm J. 2019; 23:18-293. doi:https://doi.org/10.7812/TPP/18293. Available at https://www.ncbi.nlm.nih.gov/pmc/articles/ PMC6636526/. Accessed 28 Sept 2021.

13. Department of Health and Human Services (DHHS) Notification of enforcement discretion for telehealth remote communications during COVID-19, January 20, 2021. https://www.hhs.gov/hipaa/ for-professionals/special-topics/emergency-preparedness/notificati on-enforcement-discretion-telehealth/index.html. Accessed 30 July 2021.
14. COVID-19 Emergency declaration blanket waivers for healthcare providers, CMS, August 2020. https://www.cms.gov/about-cms/ emergency-preparedness-response-operations/current-emerg encies/coronavirus-waivers. Accessed 30 July 2021.

15. DEA Guidance: How to prescribe controlled substances during COVID-19. 2020. https://www.deadiversion.usdoj.gov/coronavirus.html. Accessed 30 July 2021.

16. Ramtekkar U, Bridge J, Thomas G, Butter E, Reese J, Logan E, Lin S, Axelson D. Pediatric telebehavioral health: a transformational shift in care delivery in the era of COVID-19. JMIR Ment Health. 2020;7(8):e20157. https://doi.org/10.2196/20157.

17. Sharma A, Sasser T, Schoenfelder Gonzalez E, Vander Stoep A, Myers K. Implementation of home-based telemental health in a large child psychiatry department during the COVID-19 crisis. J Child Adolesc Psychopharmacol. 2020;30:404-13.

18. Shore J, Schneck C, Mishkind MC. Telepsychiatry and the coronavirus disease 2019 pandemic - current and future outcomes of the rapid virtualization of psychiatric care. JAMA Psychiat. 2020;77(12):1211-2. https://doi.org/10.1001/jamapsychiatry. 2020.1643.

19. Folk JB, Schiel MA, Oblath R, Feuer V, Sharma A, Khan S, et al. The transition of academic mental health clinics to telehealth during the COVID-19 pandemic. J Am Acad Child Adolesc Psychiatry. 2021;S0890-8567(21):00367-71.

20. Shachar C, Engel J, Elwyn G. Implications for telehealth in a postpandemic future: regulatory and privacy issues. JAMA. 2020;323(23):2375-6. https://doi.org/10.1001/jama.2020.7943.

Publisher's Note Springer Nature remains neutral with regard to jurisdictional claims in published maps and institutional affiliations. 